\title{
MAXIMAL SEPARABLE INTERMEDIATE FIELDS OF LARGE CODEGREE
}

\author{
NICKOLAS HEEREMA
}

\begin{abstract}
Let $\boldsymbol{k}$ be a function field in $n(n>0)$ variables over $k_{0}$ a field having characteristic $p \neq 0$. An intermediate field $s$ is maximal separable if $s / k_{0}$ is separable and $s$ is not properly contained in any subfield of $k$ separable over $k_{0}$. The following result is proved. If $n=1$ the set $\Delta=\{[k: s] \mid s$ maximal separable $\}$ is bounded if and only if the algebraic closure $\bar{k}_{0}$ of $k_{0}$ in $k$ is separable over $k_{0}$. If $n>1$ and $\Delta$ is bounded then $\bar{k}_{0} / k_{0}$ is separable. An upper bound for $\Delta$ is obtained for the case $n=1$ and $\bar{k}_{0} / k_{0}$ separable.
\end{abstract}

I. Introduction. In 1947 Dieudonné initiated the study of maximal separable intermediate fields [2]. Let $k \supset k_{0}$ be fields of characteristic $p \neq 0$ with $k$ finitely generated over $k_{0}$. Let $r$ be the exponent of inseparability of $k / k_{0}$, that is, $r$ is the least positive integer such that $k_{0}\left(k^{p^{r}}\right) / k_{0}$ is separable. Dieudonne showed that the maximal separable intermediate fields $s$ with the property $k \subset k_{0}^{p^{-\infty}}(s)$, which he called distinguished subfields of $k / k_{0}$, are those of the form $k_{0}\left(k^{p^{\prime}}\right)(T)$ where $T^{p^{\prime}}$ is a $p$-basis for $k_{0}\left(k^{p^{\prime}}\right) / k_{0}$. He also observed that all such fields have minimal codegree $\left[k: k_{0}\right]_{i}$ among intermediate fields separable over $k_{0}$. In $1970 \mathrm{Kraft}$ [5] showed that the distinguished subfields of $k / k_{0}$ are precisely those intermediate fields $s$ separable over $k_{0}$ for which $[k: s]$ is minimal. Dieudonné showed by example that not all maximal separable intermediate fields are distinguished. Recently Deveney and Mordeson have reported results on the characterization of those field extensions $k / k_{0}$ for which every maximal separable intermediate field is distinguished. In this note we consider a complementary question. When is $\Delta=$ $\left\{[k: s] \mid s\right.$ a maximal separable subfield of $\left.k / k_{0}\right\}$ bounded? Theorem 1 states that if the transcendency degree of $k / k_{0}$ is one then $\Delta$ is bounded if and only if the algebraic closure $\bar{k}_{0}$ of $k_{0}$ in $k$ is separable over $k_{0}$. Corollary 1 provides an upper bound for $\Delta$ if $\bar{k}_{0} / k_{0}$ is separable and Corollary 2 asserts that, dropping the restriction on transcendency degree, if $\Delta$ is bounded then $\bar{k}_{0} / k_{0}$ is separable. We conjecture the converse having been unable to settle the matter.

The fields $k_{0}\left(k^{(n)}\right)=\left\{a \in k \mid\right.$ for some $\left.t>0, a^{p^{t}} \in k_{0}\left(k^{p^{t+n}}\right)\right\}$ have an essential role in this paper. Their basic properties are investigated in [3].

II. Maximal separable fields. Throughout $k \supset k_{0}$ are fields of characteristic $p \neq 0$ with $k$ finitely generated over $k_{0}$. The exponent of inseparability of $k / k_{0}$ is $r$. The abbreviation tr.deg. $\left(k / k_{0}\right)$ denotes the transcendency degree of $k / k_{0}$. We begin with a statement (A) of basic and familiar facts.

Received by the editors June 11, 1980.

1980 Mathematics Subject Classification. Primary 12F15, 12 F20. 
(A) If $k / k_{0}$ is separable then (i) $k / k_{0}$ is separably generated and (ii) a $p$-basis for $k / k_{0}$ is a separating transcendency basis for $k / k_{0}$ and conversely.

The definition of a separable extension, namely $s / k_{0}$ is separable if $s$ and $k_{0}^{p^{-1}}$ are linearly disjoint over $k_{0}$, implies that if $U$ is a linear basis for $s / k_{0}$ then $U^{p^{n}}$ is a linear basis for $k_{0}\left(s^{p^{n}}\right) / k_{0}$ from which fact one derives the following.

(B) If $a^{p^{r}} \in k_{0}\left(k^{p^{n+r}}\right) \backslash k_{0}\left(k^{p^{n+r+1}}\right)$ then $a \in k_{0}\left(k^{(n)}\right) \backslash k_{0}\left(k^{n+1}\right)$ and conversely.

We shall also use the following result.

(C) [4, Lemma 2.4] An intermediate field $s$ such that $s / k_{0}$ is separable and $k / s$ is radical is a maximal separable intermediate field if and only if $k^{p} \cap s \subseteq k_{0}\left(s^{p}\right)$.

Proposition 1. If tr.deg. $\left(k / k_{0}\right)=1$ and $s$ is an intermediate field separable over $k_{0}$ with $k / s$ a radical extension then, for some integer $q, q>0, k_{0}\left(s^{p^{\prime}}\right)=k_{0}\left(k^{p^{r+q}}\right)$. Also, the following are equivalent:

(i) $k_{0}\left(s^{p^{\prime}}\right)=k_{0}\left(k^{p^{r+q}}\right)$.

(ii) $s \subset k_{0}\left(k^{(q)}\right) \backslash k_{0}\left(k^{(q+1)}\right)$.

(iii) $[k: s]=p^{q}\left[k: k_{0}\right]_{i}$.

Proof. Since $k / s$ is finitely generated radical $k_{0}\left(k^{p m}\right) \subset s$ for some $m>0$. Thus $k_{0}\left(k^{p^{m+r}}\right) \subset k_{0}\left(s^{p^{\prime}}\right) \subset k_{0}\left(k^{p^{\prime}}\right)$. Since tr.deg. $\left(k / k_{0}\right)=1$ and $k_{0}\left(k^{p^{\prime}}\right) / k_{0}$ is separable $k_{0}\left(k^{p^{\prime}}\right)$ has a one element $p$-basis over $k_{0}$ and thus $k_{0}\left(k^{p^{\prime}}\right)$ is a simple extension of $k_{0}\left(k^{p^{r+m}}\right)$. It follows that the only intermediate fields are those of the form $k_{0}\left(k^{p^{q+r}}\right), 0 \leqslant q \leqslant m$, and so $k_{0}\left(s^{p^{\prime}}\right)=k_{0}\left(k^{p^{q+r}}\right)$ for some $q$.

Assume (i). By statement (B), $s \subset k_{0}\left(k^{(q)}\right) \backslash k_{0}\left(k^{(q+1)}\right)$. Conversely, if $s \subset$ $k_{0}\left(k^{(q)}\right) \backslash k_{0}\left(k^{(q+1)}\right)$ then, by (B), $k_{0}\left(s^{p^{\prime}}\right) \subset k_{0}\left(k^{p^{r+q}}\right) \backslash k_{0}\left(k^{p^{r+q+1}}\right)$. Hence $k_{0}\left(s^{p^{\prime}}\right)=$ $k_{0}\left(k^{p^{r+q}}\right)$.

To show that (i) implies (iii) let $s_{1}$ be a distinguished subfield of $k / k_{0}$. Then $k_{0}\left(s_{1}^{p^{\prime}}\right)=k_{0}\left(k^{p^{\prime}}\right)$ [5, Satz III, p. 113] and, assuming (i),

$$
\begin{aligned}
{\left[k: k_{0}\left(k^{p^{r+q}}\right)\right] } & =\left[k: s_{1}\right]\left[s_{1}: k_{0}\left(s_{1}^{p^{\prime}}\right)\right]\left[k_{0}\left(s_{1}^{p^{\prime}}\right): k_{0}\left(k^{p^{r+q}}\right)\right] \\
& =[k: s]\left[s: k_{0}\left(s^{p^{\prime}}\right)\right] .
\end{aligned}
$$

By statement (A), $p$-bases for $s$ and $s_{1}$ over $k_{0}$ have the same cardinality, namely one, and hence $\left[s_{1}: k_{0}\left(s_{1}^{p^{\prime}}\right)\right]=\left[s: k_{0}\left(s^{p^{\prime}}\right)\right]$. Thus $[k: s]=\left[k: k_{0}\right]_{i} p^{q}$. Conversely, if (iii) then, by (1), $\left[k: k_{0}\left(k^{p^{r+q}}\right)\right]=\left[k: k_{0}\left(s^{p^{\prime}}\right)\right]$ and since $k_{0}\left(s^{p^{\prime}}\right)=k_{0}\left(k^{p^{\prime+m}}\right)$ for some $m$ it must be that $k_{0}\left(s^{p^{r}}\right)=k_{0}\left(k^{p^{r+q}}\right)$.

Proposition 2. Assume that tr.deg. $\left(k / k_{0}\right)=1$. If $k_{0}\left(k^{(q)}\right) \not k_{0}\left(k^{p}\right)$ there is a maximal separable intermediate field $s$ with the property $s \subset k_{0}\left(k^{(q)}\right) \backslash k_{0}\left(k^{(q+1)}\right)$.

Proof. Let $a$ be in $k_{0}\left(k^{(q)}\right) \backslash k_{0}\left(k^{p}\right)$. If $a$ is in $k_{0}\left(k^{(q+1)}\right)$ replace $a$ by $a+b^{p^{q}}$ where $b \in s_{1} \backslash k_{0}\left(s_{1}^{p}\right)$ for a distinguished subfield $s_{1}$ of $k / k_{0}$. Then by statement (B), the fact that $b^{p^{\prime}} \in k_{0}\left(s_{1}^{p^{\prime}}\right) \backslash k_{0}\left(s_{1}^{p^{r+1}}\right)$ and noting that $k_{0}\left(s_{1}^{p^{\prime}}\right)=k_{0}\left(k^{p^{\prime}}\right)$ we have $b^{p^{q}} \in k_{0}\left(k^{(q)}\right) \backslash k_{0}\left(k^{(q+1)}\right)$. Thus we can assume that $a \in k_{0}\left(k^{(q)}\right) \backslash k_{0}\left(k^{(q+1)}\right)$ and $a \notin k_{0}\left(k^{p}\right)$. Let $s=k_{0}\left(k^{p^{r+q}}\right)(a)$. Since $a^{p^{r}} \in k_{0}\left(k^{p^{r+q}}\right) \backslash k_{0}\left(k^{p^{r+q+1}}\right)$, $a^{p^{r}}$ is a separating transcendency basis for $k_{0}\left(k^{p^{r+q}}\right) / k_{0}$. It follows that $s / k_{0}$ is separable. Clearly $k / s$ is radical. If $s$ is not maximal separable then, by statement (C), $k^{p} \cap s \not \subset k_{0}\left(s^{p}\right)$. Hence there is an element $b$ in $k$ such that $b^{p} \in s \backslash k_{0}\left(s^{p}\right)$. Then 
$\left\{b^{p}\right\}$ is a $p$-basis for $s / k_{0}$ and $s=k_{0}\left(s^{p}\right)\left(b^{p}\right) \subset k_{0}\left(k^{p}\right)$ contradicting the fact that $a$ is in $s$. Thus $s$ is maximal separable and, by construction, $s \subset k_{0}\left(k^{(q)}\right)$.

Proposition 3. Assume tr.deg. $\left(k / k_{0}\right)=1$. Let $s$ be a maximal separable subfield of $k_{0}\left(k^{(q)}\right)$ contained in $k_{0}\left(k^{(q+t)}\right)$. If $s \subset s_{1}$ and $s_{1}$ is a maximal separable subfield of $k / k_{0}$ then $\left[k: s_{1}\right] \geqslant p^{t}\left[k: k_{0}\right]_{i}$.

Proof. Let $k_{1}=k_{0}\left(k^{(q)}\right)$. Then $k_{0}\left(k^{(q+t)}\right)=k_{0}\left(k_{1}^{(t)}\right)$ [3, Lemma 4, p. 289] and so, by Proposition $1,\left[k_{1}: s\right]>p^{t}\left[k_{1}: k_{0}\right]_{i}$. Since $s_{1}^{p^{q}} \subset k_{1}$ it follows that $k_{0}\left(s_{1}^{p^{n}}\right) \subset s$ for some $n$. Hence $s_{1}=s(a)$ for some $a$ and $a^{p^{q}}$ is in $k_{1}$. Since $s\left(a^{p^{q}}\right)$ is separable over $k_{0}$ and $s$ is maximal separable in $k_{1}$ it follows that $a^{p^{q}}$ is in $s$. Thus $\left[s_{1}: s\right]<p^{q}$ and $\left[k: s_{1}\right]=\left[k: k_{1}\right]\left[k_{1}: s\right] /\left[s_{1}: s\right]>\left[k: k_{1}\right]\left[k_{1}: k_{0}\right]_{i} p^{t-q}$. If $s_{2}$ is a distinguished subfield of $k / k_{0}$ then $k_{0}\left(s_{2}^{p^{q}}\right)$ is a distinguished subfield of $k_{1}$ [3, Theorem 2, p. 288]. Thus, $\left[k: k_{0}\right]_{i}\left[s_{2}: k_{0}\left(s_{2}^{p^{q}}\right)\right]=\left[k: k_{1}\right]\left[k_{1}: k_{0}\right]_{i}$ or $\left[k: k_{0}\right]_{i} p^{q}=$ $\left[k_{1}: k_{0}\right]_{i}\left[k: k_{1}\right]$. Substituting this in the above inequality yields $\left[k: s_{1}\right]>$ $\left[k: k_{0}\right]_{i} p^{t}$.

Theorem 1. If tr.deg. $\left(k / k_{0}\right)=1$ the set $\Delta=\{[k: s] \mid s$ a maximal separable subfield of $\left.k / k_{0}\right\}$ is bounded if and only if the algebraic closure of $k_{0}$ in $k$ is $a$ separable extension of $k_{0}$.

Proof. Let $\bar{k}_{0}$ be the algebraic closure of $k_{0}$ in $k$. Let $k_{1}=k_{0}\left(k^{(q)}\right)$ where $q$ is chosen large enough so that $k_{0}\left(k^{(q)}\right)=\bar{k}_{0}\left(k^{p^{q}}\right)$ and $k_{0}\left(k^{p^{q}}\right) / k_{0}$ is separable [3, Theorem 9, p. 290]. If $\bar{k}_{0} / k_{0}$ is inseparable $\bar{k}_{0}$ has a nonempty $p$-basis $T$ over $k_{0}$. Since $k_{0}\left(k^{p^{q}}\right) / k_{0}$ is separably generated $\bar{k}_{0}\left(k^{p^{q}}\right) / \bar{k}_{0}$ is separable. Separable extensions are $p$-independence preserving so $T$ is a $p$-independent subset of $k_{1} / k_{0}$. Since $\bar{k}_{0} \subset k_{0}\left(k^{(q+t)}\right)=k_{0}\left(k_{1}^{(t)}\right)$ for all $t$ [3, Lemma 4, p. 289] it follows that $T \subset k_{0}\left(k_{1}^{(t)}\right)$ for all $t$ and we have $k_{0}\left(k_{1}^{(t)}\right) \not \subset k_{0}\left(k_{1}^{p}\right)$ for all $t>0$. By Proposition 2, $k_{1}$ contains a maximal separable subfield $s$ over $k_{0}$ which is in $k_{0}\left(k_{1}^{(t)}\right)$. By Proposition 3, if $s_{1}$ is a maximal separable subfield of $k / k_{0}$ containing $s$ then $\left[k: s_{1}\right]>\left[k: k_{0}\right]_{i} p^{t}$. This establishes the result in one direction since $t$ can be chosen arbitrarily large.

To show the converse we note that if $\bar{k}_{0} / k_{0}$ is separable then, for large $q$, $k_{0}\left(k^{(q)}\right) / k_{0}=\bar{k}_{0}\left(k^{p^{q}}\right) / k_{0}$ is separable. Thus, if $s$ is a maximal separable subfield of $k / k_{0}$ and $[k: s]=\left[k: k_{0}\right]_{i} p^{q}$ for $q$ as above then, by Proposition $1, s \subset k_{0}\left(k^{(q)}\right)$ and so $s=k_{0}\left(k^{(q)}\right)$. This leads to a contradiction since $\bar{k}_{0}\left(k^{p^{q}}\right)$ is contained in every distinguished subfield. It follows that $[k: s]<\left[k: k_{0}\right]_{i} p^{n}$ where $n$ is the least integer $j$ such that $k_{0}\left(k^{(j)}\right)=\bar{k}_{0}\left(k^{p^{j}}\right)$ and $k_{0}\left(k^{p^{j}}\right) / k_{0}$ is separable.

The following remarks do not require the assumption tr.deg. $\left(k / k_{0}\right)=1$. If $s$ is a distinguished subfield of $k / k_{0}$ then $k_{0}\left(s^{p^{n}}\right)$ is a distinguished subfield of $k_{0}\left(k^{(n)}\right)$ and, so, if $k_{0}\left(k^{(n)}\right) / k_{0}$ is separable, $k_{0}\left(s^{p^{n}}\right) \subset k_{0}\left(k^{p^{n}}\right) \subset k_{0}\left(k^{(n)}\right)=k_{0}\left(s^{p^{n}}\right)$. Thus, $k_{0}\left(k^{(n)}\right)=\bar{k}_{0}\left(k^{p^{n}}\right)=k_{0}\left(k^{p^{n}}\right)$ since $\bar{k}_{0} \subset k_{0}\left(k^{(n)}\right)$ for all $n$ [3, Theorem 5, p. 289]. Thus $k_{0}\left(k^{(n)}\right) / k_{0}$ is separable for some $n$ if and only if $\bar{k}_{0} / k_{0}$ is separable, and if $k_{0}\left(k^{(n)}\right) / k_{0}$ is separable then $k_{0}\left(k^{(n)}\right)=k_{0}\left(k^{p^{n}}\right)$.

COROLlaRY 1. If tr.deg. $\left(k / k_{0}\right)=1$ the following are equivalent.

(i) $k_{0}\left(k^{(n)}\right) / k_{0}$ is separable for some $n$.

(ii) $\bar{k}_{0} / k_{0}$ is separable. 
(iii) $\left\{[k: s] \mid s\right.$ a maximal separable subfield of $\left.k / k_{0}\right\}$ is bounded.

If one of the three conditions holds and $s$ is a maximal separable subfield of $k / k_{0}$ then $[k: s]<\left[k: k_{0}\right]_{i} p^{n}$ where $n$ is the least integer such that $k_{0}\left(k^{(n)}\right) / k_{0}$ is separable.

The following result holds for arbitrary transcendence degree $>0$.

COROLLARY 2. If $\bar{k}_{0} / k_{0}$ is inseparable then $\{[k: s] \mid s$ a maximal separable subfield of $\left.k / k_{0}\right\}$ is unbounded.

Proof. ${ }^{1}$ Let $\left\{x_{1}, \ldots, x_{l}\right\}$ be a transcendency basis for a distinguished subfield of $k / k_{0}$. Replace $k_{0}$ with $k_{1}=k_{0}\left\{x_{1}, \ldots, x_{l-1}\right\}$. We assume $l>1$ having the result for $l=1$. If $\bar{k}_{0} / k_{0}$ is inseparable then $\bar{k}_{1} / k_{1}$ is inseparable and $\{[k: s] \mid s$ is a maximal separable subfield of $\left.k / k_{1}\right\}$ is unbounded by Theorem 1 . It remains to show that if $s$ is a maximal separable subfield of $k / k_{1}$ it is a maximal separable subfield of $k / k_{0}$. By [4, Proposition 2.4] we have $k^{p} \cap s \subset k_{1}\left(s^{p}\right)$ or $k^{p} \cap s \subset$ $k_{1}\left(s^{p}\right) \cap k^{p}=k_{0}\left(x_{1}, \ldots, x_{l-1}\right)\left(s^{p}\right) \cap k^{p}$. Since $\left\{x_{1}, \ldots, x_{l-1}\right\}$ is a $p$-independent subset of $k / k_{0}$ it follows that $k^{p} \cap s \subset k_{0}\left(x_{1}^{p}, \ldots, x_{l-1}^{p}\right)\left(s^{p}\right) \subset k_{0}\left(s^{p}\right)$. Hence, by statement (C), $s$ is a maximal separable subfield of $k / k_{0}$.

\section{REFERENCES}

1. J. Deveney and J. Mordeson, Distinguished subfields, preprint.

2. J. Dieudonné, Sur les extensions transcendantes, Summa Brasil. Math. 2 (1947), 1-20. MR 10, 5.

3. N. Heerema, pth powers of distinguished subfields, Proc. Amer. Math. Soc. 55 (1976), 287-291.

4. N. Heerema and T. Morrison, Maximal separable subfields of finitely generated extensions, preprint.

5. H. Kraft, Inseparable Körpererweiterungen, Comment. Math. Helv. 45 (1970), 110-118. MR 41 \#5333.

Department of Mathematics, Florida State University, Tallahasseg, Florida 32306

\footnotetext{
1 The referee has observed that Corollary 2 can be proved in a way which avoids the use of Propositions 2 and 3 by first proving the following. Proposinon. If $k / k_{0}$ is finitely generated and has maximal separable subfields of unbounded codimension the same is true for any finite extension of $k$.

Corollary 2 is then reduced to the case $k=k_{0}\left(x_{1}, \ldots, x_{n}\right)(b)$ where the $x_{i}$ are indeterminates and $b^{p}$ is in $k_{0}$, not in $k f$.
} 